\title{
RESONANCE SPECTRA OF QUANTUM DOTS IN MAGNETIC FIELD
}

\author{
M. BYLICKI AND W. JaSKóLSKI \\ Instytut Fizyki UMK, Grudziądzka 5, 87-100 Torun, Poland
}

\begin{abstract}
Influence of magnetic field on the energy positions and widths of one-electron resonance states in a multi-shell spherical quantum dot is investigated. The one-band effective mass approximation is assumed. The complex-eigenvalue Schrödinger equation approach involving complex rotation of coordinates is used to obtain complex eigenenergies, $E_{\mathrm{r}}-\mathrm{i} \Gamma / 2$, corresponding to resonance states. We show how the magnetic field changes the resonance energy, $E_{\mathrm{r}}$, and decay rate, $\Gamma$, yielding bound states for some particular cases.
\end{abstract}

P.ACS numbers: 73.20.Dx, 73.61.-r, 85.30.Vw, 73.23.Hk

\section{Introduction}

Progress in semiconductor nanotechnology has led in the last few years to chemical synthesis of $\mathrm{CdS}, \mathrm{CdSe}$, and InAs nanocrystals of almost spherical or hemispherical shapes and of a very well-controlled size ranging from 1 to $10 \mathrm{~nm}$ [1-3]. Recently, multi-shell nanocrystals, built of several quasi-spherical layers of CdS and $\mathrm{HgS}$ of nanometer thickness, have also been synthesized [4]. The physics of such quantum dots make them extremely interesting for novel optoelectronic applications, because the internal structure of these systems can be tailored to control energy levels (transition energies) and induce electron-hole charge separation to modify their optical properties [5]. A formation of two-dimensional and three-dimensional arrays of spherical nanocrystals, recently achieved, can provide devices with enhanced optical activity [1]. Tunneling transport of carriers between such dots can also be thought as possible.

So far the resonant tunneling phenomena have been studied mainly for models of standard epitaxially grown quantum dots with quasi two-dimensional geometries [6]. The influence of magnetic field on the energy spectra of quantum dots was investigated for similar geometries and with parabolic confining potential assumed [7]. In multi-shell nanocrystals the scheme of positions of conduction band edges along the radius of dot is far from parabolic, but resembles quantum well or quantum barrier structure $[5,8,9]$. Resonant tunneling states in such systems have been recently studied by us with the use of a complex coordinate approach [9]. 
In this paper we study the influence of magnetic field on the resonance tunneling states in spherical multi-shell nanocrystals. We show how the energy positions and widths of resonant states change with an applied magnetic field $B$. We find that for some values of $B$ the resonant states undergo abrupt changes of their widths and positions leading even to bound states for some particular fields. This means that in optoelectronic devices based on multi-shell nanocrystals the magnetic field could be used to control the resonant carrier transmission. We give a brief explanation of our results and we refer to similar effects found recently for autoionizing states of $\mathrm{H}^{-}$ion in magnetic field [10].

\section{Calculation and results}

The quantum dot studied in this work consists of three shells of semiconductors of different energy gaps. The internal core, of radius equal to $7 \mathrm{~nm}$, is made of small gap $\mathrm{Ga}_{0.47} \mathrm{In}_{0.53} \mathrm{As}$ material. The middle shell consists of a $2.5 \mathrm{~nm}$ thick cap of $\mathrm{Al}_{x} \operatorname{In}_{1-x} \mathrm{As}$ with $x$ close to 0.48 in order to get $0.47 \mathrm{eV}$ difference between the bottoms of conduction band in the core and middle shell. The external macroscopic shell is again built of $\mathrm{Ga}_{y} \operatorname{In}_{1-y}$ As with $y$ yielding position of the conduction band edge $10 \mathrm{meV}$ higher than in the core. This level is assumed as the reference energy position. The effective mass $m^{*}=0.041 m_{0}$, corresponding to the $\mathrm{Ga}_{0.47} \mathrm{In}_{0.53} \mathrm{As}$ well, is assumed for the whole system. This simplification is justified by the observation of Ref. [11] that, as far as the resonance energies are considered, the effects of mass-inhomogeneity and non-parabolicity are negligible for double-barrier layered structures built of the above-mentioned materials.

One electron in such a quantum dot placed in a homogeneous static magnetic field, $B$, along the $z$-axis is described by the Hamiltonian ${ }^{\dagger} H=\left(1 / 2 m^{*}\right) \Delta+$ $V(r)+\left(1 / 8 m^{*}\right) B^{2} \rho^{2}$. The magnetic field breaks the spherical symmetry of our quantum dot. Thus the orbital angular momentum is no longer a constant of motion and the eigenstates of the Hamiltonian can be labeled by the magnetic quantum number, $m$, and parity, $\pi$. The wave function of symmetry $m^{\pi}$ can be expanded in spherical orbitals corresponding to, respectively, even or odd orbital quantum numbers $l \geq|m|$. We confine this expansion to $l \leq 9$.

The energy positions, $E_{\mathrm{r}}$, and widths, $\Gamma$, of resonance states are related to some complex eigenvalues, $E_{\mathrm{r}}-\mathrm{i} \Gamma / 2$, of the Hamiltonian. They are computed in this paper by using the complex eigenvalue Schrödinger equation method [12] which involves complex coordinates. The method, described in detail in Refs. [12, 13, 9], has been already applied to study resonances in double barrier structures [13] and quantum dots [9]. It consists in solving the Schrödinger equation for complex eigenvalues within a variational basis set divided into two subsets. One of them is responsible for description of the localized part of the resonance wave function and, in this work, consists of Slater-type orbitals (STOs). The other part of the basis set has to describe the decaying character of the resonance. It contains STOs of radial variables transformed by complex rotation: $r \rightarrow r \mathrm{e}^{-\mathrm{i} \theta}$, where $\theta$ is a real parameter. Such rotated functions (STOs) can de-

${ }^{\dagger}$ Although the linear Zeeman term is not negligible, it is omitted here because of its trivial additive character. 
scribe the asymptotic non-square-integrable part of the resonance wave function, although they are square-integrable themselves.

Thirty unrotated and fifteen rotated STOs of each spherical symmetry have been used in the present calculation. They have been chosen so that the $\langle r\rangle$ for unrotated STOs ranged within the well width, where the resonance function is expected to localize. The rotated STOs were chosen so as to cover in an analogical way the region outside the well up to $300 \mathrm{~nm}$. The Hamiltonian matrix is built and diagonalized for many values of $\theta$ ranging from 0 to 1.2 radians. The roots that stabilize against variations of $\theta$ are considered as approximate complex energies of resonant states.

\section{TABLE}

Resonances and virtual states in the field free case. The axial symmetry assignment $m^{\pi}(n)$ of the states evolving from them in a non-zero magnetic field is given (only the $m=0,1$ states investigated in this work are listed). Numbers in parenthesis label consecutive states of a given $m^{\pi}$ symmetry.

\begin{tabular}{rr|l|l|l}
\hline \multicolumn{1}{c|}{ State } & $E_{\mathrm{r}}[\mathrm{eV}]$ & $-\Gamma / 2[\mathrm{eV}]$ & Axial symmetry \\
\hline \multicolumn{2}{c|}{ even } & & & \\
& $s$ & 0.0247 & 0.0017 & $0^{\mathrm{e}}(1)$ \\
& $d$ & 0.315 & 0.020 & $0^{\mathrm{e}}(2), 1^{\mathrm{e}}(1)$ \\
& $s$ & 0.387 & 0.035 & $0^{\mathrm{e}}(3)$ \\
& $g$ & 0.725 & 0.064 & $0^{\mathrm{e}}(4), 1^{\mathrm{e}}(2)$ \\
& $d$ & 0.891 & 0.134 & $0^{\mathrm{e}}(5), 1^{\mathrm{e}}(3)$ \\
\multicolumn{1}{c|}{ odd } & & & \\
& & & & \\
& $p$ & 0.154 & 0.008 & $0^{\circ}(1), 1^{\circ}(1)$ \\
& $f$ & 0.505 & 0.038 & $0^{\circ}(2), 1^{\circ}(1)$ \\
& $p$ & 0.627 & 0.073 & $0^{\circ}(3), 1^{\circ}(1)$
\end{tabular}

The quantum dot we deal with does not have any bound state in the energy range of conduction band. It has several resonances and virtual states ${ }^{\ddagger}$. Their energies and widths found for $B=0$ are collected in Table. The influence of magnetic field on these quasi-stationary states is investigated here for the fields ranging from 0 to $70.5 \mathrm{~T}$. The results are shown in Figs. 1 and 2 for the even and odd parity states, respectively.

In all the cases shown in Figs. 1 and 2, the zero-field energy of a given state is the left-most one and moves to the right with the increasing magnetic field. This is what should be expected - the diamagnetic term of the Hamiltonian should give a positive contribution to the energy. Another characteristic property,

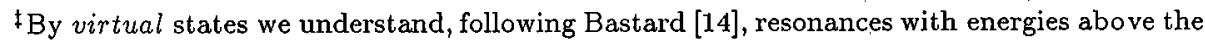
barrier.
} 


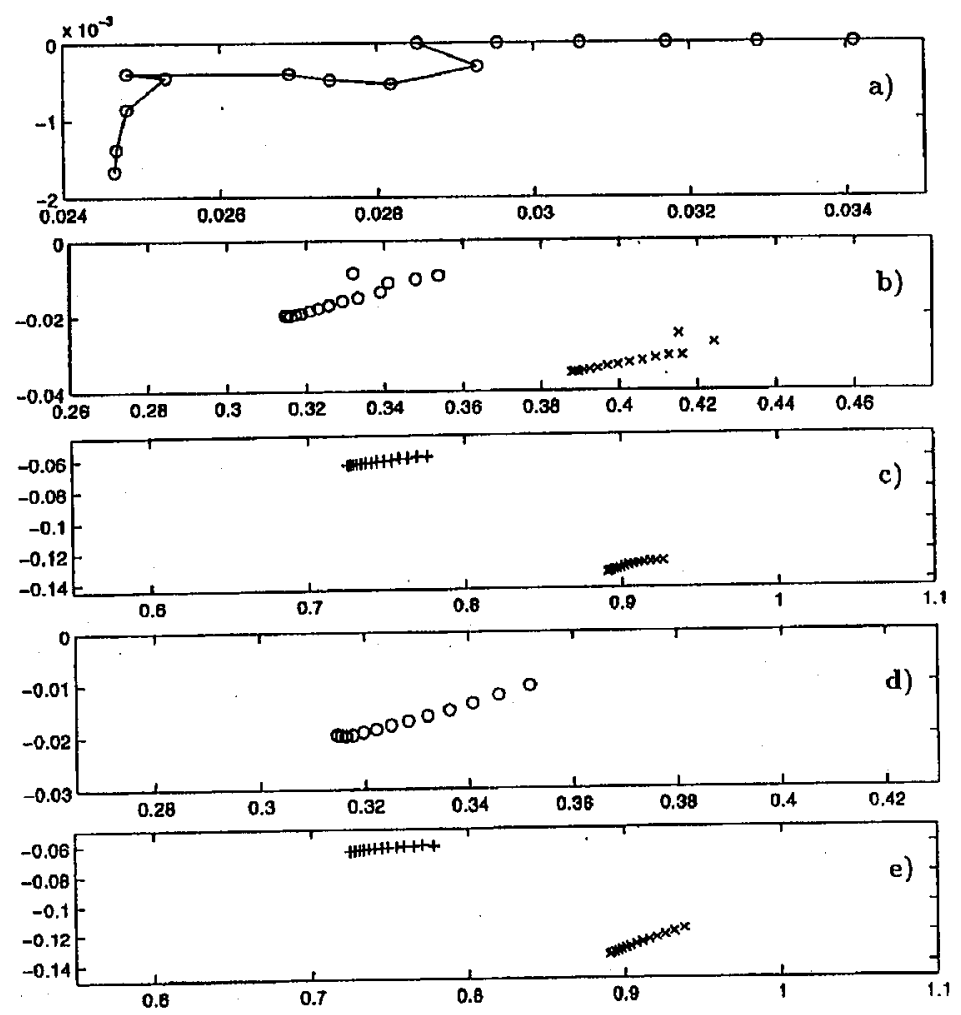

Fig. 1. Field dependence of the complex energies $E_{\mathrm{r}}-\mathrm{i} \Gamma / 2$ of the $m^{\mathrm{e}}(n)$ states, where $n$ numbers the states within a given axial symmetry $m^{\pi} . E_{\mathrm{r}}$ - horizontal and $-\mathrm{i} \Gamma / 2$ vertical axis in $\mathrm{eV}$. (a) $0^{\mathrm{e}}(1)$; (b) (o) $0^{\mathrm{e}}(2),(\times) 0^{\mathrm{e}}(3)$; (c) (+) $0^{\mathrm{e}}(4),(\times) 0^{\mathrm{e}}(5)$; (d) $1^{\mathrm{e}}(1)$; (e) $(+) 1^{e}(2),(x) 1^{e}(3)$.

common for all states, is that the widths, $\Gamma$, decrease when the field increases. This is intelligible if one realizes that the increasing magnetic field binds stronger and stronger the electron in the plane perpendicular to the field axis, leaving the field direction as the only way for the electron to tunnel out from the dot. In most of the cases this behavior is very smooth. However, for lower states one can observe sudden jumps of the complex energies.

This intriguing phenomenon can be explained as follows. The "structureless" field-free continuum splits in the magnetic field into continua associated with Landau states of free electron, i.e., the free electron energy consists of a Landau level energy, $\epsilon_{k}$, and kinetic energy of the free motion in the field axis. The Landau level energies, $\epsilon_{k}=\left(1 / 2 m^{*}\right) B(|m|+k)$, constitute continuum thresholds that change linearly with the magnetic field $B$, whereas, for small fields, the quasi-stationary state positions change more slowly. Therefore, the Landau channels become consecutively closed for the tunneling when the field increases. 


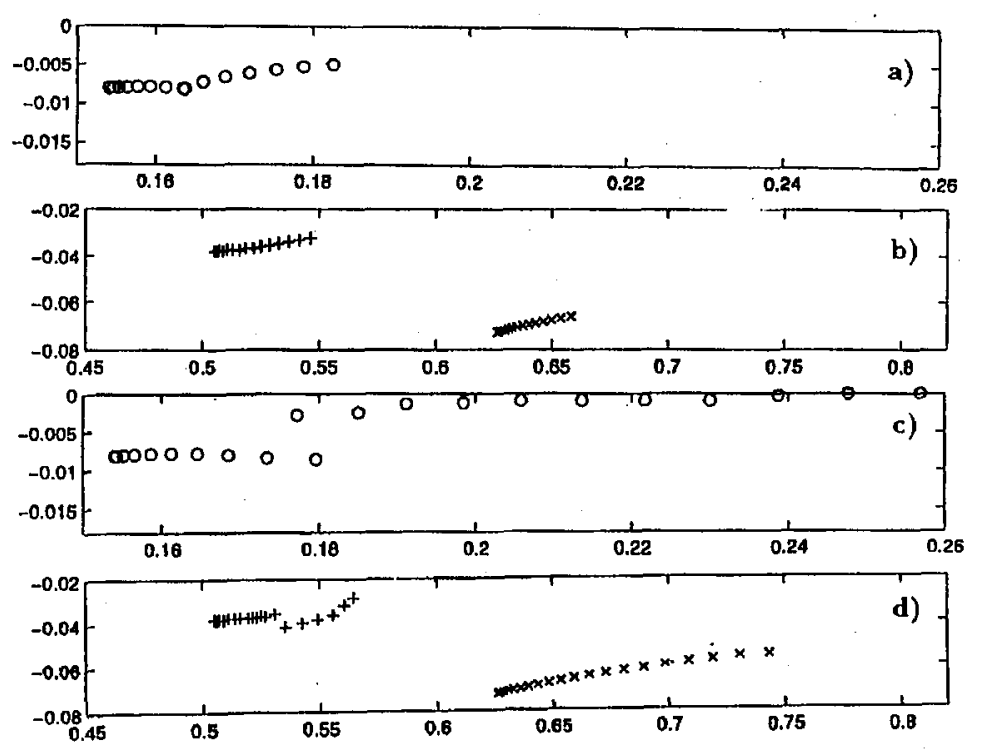

Fig. 2. Field dependence of the complex energies $E_{\mathrm{r}}-\mathrm{i} \Gamma / 2$ of the $m^{\circ}(n)$ states, where $n$ numbers the states within a given axial symmetry $m^{\circ} . E_{\mathrm{r}}-$ horizontal and $-\mathrm{i} \Gamma / 2$ — vertical axis in $\mathrm{eV}$. (a) $0^{\circ}(1)$; (b) (+) $0^{\circ}(2)$, (x) $0^{\circ}(3)$; (c) $1^{\circ}(1)$; (d) (+) $1^{\circ}(2)$, (x) $1^{\circ}(3)$.

The effect of such channel closing is most pronounced for the lowest state $0^{\mathrm{e}}(1)$, see Fig. 1a. (The field increases here by $2.35 \mathrm{~T}$ from point to point; the aim of the solid line is just to make the identification of consecutive points easier.) By the field $B=7.05 \mathrm{~T}$ (the fourth point in Fig. 1a) only two lowest Landau channels are still open. The infinite number of other channels has become already closed causing a large reduction of the resonance width. For the next field value, $B=9.4 \mathrm{~T}$, the second Landau threshold appears at $\epsilon_{2}=0.0265 \mathrm{eV}$, while the resonance energy is $E_{\mathrm{r}}=0.0248 \mathrm{eV}$. Thus, this continuum channel is closed for the tunneling. Coupling to the continuum is reduced and the electron localization becomes stronger. This manifests by a sharp decrease in the resonance energy position. The lowest Landau channel remains open up to $B=18.8 \mathrm{~T}$ (the 9 th point in Fig. 1a). Then, for $B=21.35 \mathrm{~T}$, the $0^{\mathrm{e}}(1)$ state energy becomes real, $E=0.0285 \mathrm{eV}$, lying below the lowest Landau threshold, $\epsilon_{1}=0.0299 \mathrm{eV}$. Thus, the state is completely bound and remains bound for higher fields. Similar behavior can be seen in Fig. $2 \mathrm{c}$ for the $1^{\circ}(1)$ state, which is definitely bound for fields above $90 \mathrm{~T}$. Recently, a similar field-dependence of the energies of autoionizing shape resonance of $\mathrm{H}^{-}$ion has been also reported [10].

In conclusion, the magnetic field influence on the positions and widths of resonance states in multi-shell quantum dots has been studied. It has been shown that 
the diamagnetic term of the Hamiltonian rises the energies of resonant states and slightly decreases their widths. For some particular values of magnetic field, i.e., when the energy of a consecutive Landau level approaches the energy of a resonant state, the latter may undergo the reduction of its width yielding an increase in the tunneling time of electrons out of the quantum dot. This means that magnetic field could be used, to some extent, to control the resonance tunneling rate and thus, to control transport of carriers in optoelectronic devices based on multi-shell nanocrystals. A resonance with the energy close to the continuum threshold may become even bound. In such a case the magnetic field would act as a switch of the current conducted through the resonant state.

\section{References}

[1] D.J. Norris, M.G. Bawendi, Phys. Rev. B 53, 16338 (1996).

[2] U. Banin, J.C. Lee, A.A. Guzelian, A.V. Kadavanish, A.P. Alivisatos, W. Jaskólski, G.W. Bryant, Al.L. Efros, M. Rosen, J. Chem. Phys. 109, 2306 (1998).

[3] M.A. Anderson, S. Gorer, R.M. Penner, J. Phys. Chem. B 101, 5895 (1997).

[4] A. Mews, A.V. Kadavanich, U. Banin, A.P. Alivisatos, Phys. Rev. B 53, R13242 (1996).

[5] W. Jaskólski, G.W. Bryant, Phys. Rev. B 57, R4237 (1998).

[6] G.W. Bryant, Phys. Rev. B 39, 3145 (1989).

[7] N.F. Johnson, J. Phys., Condens. Matter 7, 965 (1995).

[8] G.W. Bryant, Phys. Rev. B 52, R16997 (1995).

[9] W. Jaskólski, M. Bylicki, Vacuum 48, 235 (1997).

[10] M. Bylicki, C.A. Nicolaides, unpublished.

[11] J.A. Porto, J. Sanchez-Dehesa, L.A. Cury, A. Nogaret, J.C. Portal, J. Phys., Condens. Matter 6, 887 (1994).

[12] C.A. Nicolaides, in: Applied Many-Body Methods in Spectroscopy and Electronic Structure, Ed. D. Mukherjee, Plenum, New York 1992, p. 233.

[13] M. Bylicki, W. Jaskólski, R. Oszwałdowski, J. Phys., Condens. Matter 8, 6393 (1996).

[14] G. Bastard, Wave Mechanics Applied to Semiconductor Heterostructures, Les Editions de Physique, Paris 1990, p. 10. 